\title{
ACTITUDES AMBIENTALES HACIA EL AGUA, UNA EXPLORACIÓN EN ESTUDIANTES DEL MUNICIPIO DE VENTAQUEMADA (BOYACÁ) ${ }^{1}$
}

\author{
Milena Paola Mendieta Hernández ${ }^{2}$ \\ Gloria Leonor Gutiérrez Gómez ${ }^{3}$
}

Recibido el 11 de julio de 2013 y aprobado el 10 de enero de 2014, actualizado el 6 junio de 2014

\section{RESUMEN}

Este artículo presenta los resultados de la primera fase de una investigación concluida y orientada a diseñar, implementar y evaluar una estrategia didáctica que promueva actitudes de conocimiento y conservación hacia el agua en estudiantes de una institución educativa rural del departamento de Boyacá (Colombia) usando como eje mediático la cosmovisión del Pueblo Muisca alrededor del agua. Para lograr tal propósito, y siguiendo los criterios de la investigación acción, se diseñó un libro código que compilaba categorías, cuestiones, ítems e intencionalidad de una serie de preguntas. Con base en este se elaboró, y sometió a proceso de validez, un cuestionario de actitudes con preguntas de selección múltiple con única respuesta y semiabiertas que permitieron valorar la dimensiones conceptual, procedimental y axiológica del constructo actitud hacia el agua entendida desde la visión compleja del ambiente. Posterior a ello se realizó un análisis del instrumento diagnóstico con el software estadístico SPSS versión 17 para las preguntas de selección múltiple y el software Atlas.ti, para las preguntas semiabiertas. Una vez analizados los resultados, se obtuvo que existe escasa correlación entre lo que se escribe, se siente y se hace respecto al agua. Dentro de las conclusiones, se insta a que el diagnóstico de actitudes sea factor precedente de estrategias didácticas que favorezcan el desarrollo de actitudes ambientalmente sustentables hacia el agua.

\section{PALABRAS CLAVE}

Actitud, agua, educación ambiental, valoración, Muiscas.

\section{ENVIRONMENTAL ATTITUDES TOWARD WATER, AN EXPLORATION IN STUDENTS FROM THE VENTAQUEMADA MUNICIPALITY (BOYACÁ)}

\begin{abstract}
This article presents the results of the first phase of an investigation completed and oriented to design, implement and evaluate a teaching strategy that promotes knowledge and conservation attitudes toward water in students from a rural school in the department of Boyacá (Colombia) using as media hub the Muisca People's world view around water. To achieve this purpose, following the criteria of action research, a code book that compiled categories, issues, items and intentionality of
\end{abstract}


a series of questions was designed. Based on this, an attitude questionnaire with multiple choice questions with only one possible answer and semi-open questions which allowed assessing the conceptual, procedural and axiological dimensions of the attitude towards water understood from the complex vision of environment was designed and submitted to validity process. Subsequently, an analysis of the diagnostic instrument was performed with SPSS Statistical Software version 17 for multiple choice questions and Atlas.ti Software, for semi-open questions. After analyzing the results, it was found that there is little correlation between what is written, what is felt and what is done on water. Among the conclusions, it is urged that the diagnosis of attitudes is precedent factor of teaching strategies that support the development of environmentally sustainable attitudes toward water.

\section{KEY WORDS}

Attitude, water, environmental education, assessment, Muiscas.

\section{INTRODUCCIÓN}

En el mundo contemporáneo son cada vez más crecientes y álgidos los problemas ambientales que aquejan a la sociedad. Ante tal situación la indiferencia de las masas de gente, que era un factor común en tiempos anteriores, se ha transformado en una evidente preocupación y conciencia del deterioro natural que sufre el planeta (Páramo y Gómez, 1997). Sin embargo, tal preocupación está centrada en una dimensión biológica y tangible del ecosistema y ciertamente faltan estudios que den cuenta de las percepciones de la comunidad frente a los matices socio-culturales de un elemento que hoy día es el nuevo oro de la humanidad: el agua.

El caso colombiano es muy particular, en específico el del departamento de Boyacá. Este se precia de ser un territorio con gran riqueza hídrica donde es evidente la disponibilidad natural del agua, representada en numerosos sistemas hídricos lénticos y lóticos de importancia nacional como los ríos Magdalena, Suárez, Chicamocha, Arauca y Meta y subcuencas de los ríos Guavio, Cravo Sur, Lengupá, Upía, Cusiana y Pauto (Gobernación de Boyacá, 2012). Pese a tal abundancia de agua, Boyacá se soporta en un sistema hídrico severamente deteriorado por los altos índices de contaminación con pesticidas y la desecación de sistemas de agua para privilegiar la agricultura, originando la reducción del sistema hídrico superficial.

Producto de lo anterior, municipios del departamento, han incluido el agua como eje ordenador en sus Planes de Ordenamiento Territorial. Lo anterior sin duda alguna ha generado cambios positivos en cuanto al mantenimiento, expansión y disponibilidad del agua como recurso, pero no ha impactado, en la mayoría de los casos, en la conservación de las 
fuentes hídricas, ni mucho menos ha contribuido en el proceso de adquirir una cultura de respeto e identidad hacia el agua entre sus pobladores.

Por tales motivos surge el proyecto de investigación denominado "Agua Viva, un legado de nuestra cultura Muisca", orientado a consolidar actitudes pro-ambientales hacia el agua al entenderla desde la visión sistémica del ambiente y como herencia ancestral de una cultura que aún tiene mucho que enseñar a propósito de la relación entre el agua y el hombre: los Muiscas.

En el presente artículo se discuten los resultados de la Fase inicial de la investigación cuyo objetivo es caracterizar las actitudes que hacia el agua tienen un grupo de estudiantes de una institución educativa rural en el departamento de Boyacá.

Un estudio de estas características es una verdadera necesidad, teniendo en cuenta que los problemas ambientales tienen fundamento en una crisis de valores donde la incorporación de actitudes ambientales es una necesidad urgente, aún más cuando el planeta está atravesando por una crisis general a causa del agua; donde las sequías e inundaciones son el comentario diario de los medios de información, que, producto de campañas gubernamentales, lanzan mensajes tergiversados que apelan por el cuidado del agua como recurso, desconociendo que es un sistema vivo y dinámico en el que se desarrollan múltiples relaciones de co-dependencia.

\section{El constructo actitud: panorama complejo}

Existe una imprecisión conceptual sobre lo que abarca el constructo de actitud, sin embargo, según Schibeci (citado por Escudero, 1985) se sabe que la actitud es un factor predecible de conducta. Es más, siguiendo a Sarabia (citado por Caurín y Gil, 2011), las actitudes son formas de expresar valores implícitos en una persona, esto al entender que el termino actitud subyace en una serie de funciones psicológicas de naturaleza motivacional.

La valoración de actitudes resulta compleja al entender, por una parte, que las actitudes son manifestaciones no observables directamente, de tal suerte que es necesario inferirlas a través de una evaluación subjetiva de las respuestas de los sujetos frente a una situación dada (Caurín y Gil, 2011), y por otra parte que se debe estudiar desde sus dimensiones cognitiva, procedimental y afectiva (Escudero, 1985; Caurín y Gil, 2011). Es más, no es posible medir una actitud específica, sino más bien algunos rasgos de ella que permiten predecir el constructo de actitud de un individuo. Así, una actitud observable hacia un fenómeno, debería estar expresada en los sentimientos, el conocimiento y la tendencia a actuar sobre el fenómeno a estudiar. 


\section{La Educación Ambiental y su relación con las actitudes}

Ciertamente el estudio de actitudes ambientales es un campo espinoso, complejo y joven en Latinoamérica, sin embargo es de común acuerdo que la Educación Ambiental (EA) se vislumbra como uno de los procesos llamados a la consolidación de las mismas.

La apuesta de una EA mediática y conciliadora entre las actitudes y las conductas de los seres humanos hacia el ambiente, se ha concebido desde hace algunos años. En 1975, en el Seminario Internacional de EA en Belgrado se propuso el término de EA como una herramienta metodológica centrada en la adopción de actitudes frente al entorno que nos rodea. Esta definición sumada al trazo de objetivos y metas sobre los que debe girar la EA se compiló en la Carta de Belgrado, que entre otros aspectos proponía que los logros en materia de EA, giraran en 5 dimensiones, a saber: conciencia, conocimiento, cambio de actitudes, capacidad de evaluación y participación (Castanedo, 1995). En la Conferencia Intergubernamental de EA en Tbilisi, en 1977, se planteó que los objetivos de la EA deberían atender a la toma de conciencia y al desarrollo de actitudes (UNESCO, 1980).

En el Congreso Internacional sobre Formación y EA en Moscú en 1987, se concibió la EA como un proceso continuo que facilita la toma de conciencia y la adquisición de valores a favor de resolver una problemática ambiental.

Vemos entonces cómo la EA lejos de ser una utopía, es un proceso con múltiples facetas llamadas todas a la modificación del comportamiento de la persona en su dimensión cognitiva y afectiva (Castanedo, 1995). Es claro que el cambio de conductas y comportamientos no es un hecho fácil ni mucho menos espontáneo, sino que avanza como parte de una intención colectiva que entiende que sobre toda actuación sobrevienen repercusiones, y que evidencia la necesidad de modificar hábitos a fin de re-significar las relaciones del ser humano con su ambiente.

\section{Antecedentes al diagnóstico de actitudes ambientales}

Acontecimientos históricos mundiales asociados a problemas álgidos como las crisis energéticas, las tensiones entre países, la inequidad de género, la creciente demanda alimentaria, entre otros, permearon los estudios en psicología desencadenando las primeras iniciativas por conceptuar las conductas a favor del ambiente o pro-ambientalistas (Aragonés y Amérigo, 1991), lo que dio origen a los primeros estudios en Psicología Ambiental.

Según Zimmermann (1995), la psicología ambiental es un campo de estudio relativamente joven, cuyos primeros cimientos se ubican hacia comienzos de la década de los sesenta, y actualmente agrupa estudios de comportamiento hacia las relaciones hombre-ambiente, partiendo de una hipótesis de 
reciprocidad en la cual el medio actúa sobre el hombre y este a su vez actúa sobre el medio.

Siguiendo a Aragonés y Amérigo (1991) el creciente interés social por el diagnostico de conductas ambientales sigue dos corrientes: una, los estudios que responden a la conducta como tal $y$, la otra, los estudios que contemplan la perspectiva actitudinal. Esta última tendencia es centro de polémicas, más aún cuando se cuestiona sobre si la actitud puede o no determinar la conducta futura de una persona, o más bien da cuenta de una percepción generalizada sobre la preocupación ambiental. No obstante, se reconoce que el diagnóstico del constructo actitud es un primer gran paso en el reconocimiento de las predisposiciones que tiene una comunidad a actuar de una manera determinada.

\section{Medición de actitudes ambientales}

Si lo que se quiere, como fin último en un estudio de carácter ambiental, es modificar o instar nuevas actitudes proambientalistas, es necesario indagar sobre las actitudes iniciales de las personas frente al problema ambiental. En la medición de actitudes ambientalistas se hace uso de escalas o cuestionarios. Existen muchas escalas para evaluar actitudes entre estas: el escalograma de Guttman, Likert, la técnica Thurstone, diferencial semántico, siendo las tipo Likert, las más usadas (Escudero, 1985). Al respecto es pertinente traer a colación los trabajos de Weigel y Weigel (1978), Van Liere y Dunlap (1981), Iwatta (1990), entre otros.

Van Liere y Dunlap (1981) construyeron un referente interesante de una escala tipo Likert de medición de actitudes ambientales, formada por varias subescalas y que permitía relacionar temas que reflejan la preocupación ambiental con variables sociodemográficas como el nivel de estudios o la ideología política. La principal crítica a la escala radicó en que dichos temas fueron producto del empirismo, dejando en entredicho su correlación. Posteriormente se fue perfeccionando de un solo ítem a respuestas multi-ítem permitiendo mayor complejidad en el análisis estadístico.

Otro referente importante es la escala de preocupación ambiental ECS (Environmental Concern Scale) propuesta por Weigel y Weigel ( 1978), que resulta importante porque los "temas sustantivos" en los que se basa son la contaminación y la conservación del ambiente. Esta escala se ha venido adaptando a fin de comprobar su validez en trabajos como el de Aragonés y Amérigo (1991).

Castanedo (1995), ajusta algunos ítems de las escalas antes mencionadas y las adapta al contexto y tiempo, además incorpora nuevos ítems obteniendo la Escala de Actitudes ProAmbientales (EAPA) de 50 ítems con puntuaciones desde muy de acuerdo a muy en desacuerdo y que mide principalmente tres aspectos: reducción de los niveles de contaminación, 
participación en la resolución de problemas medioambientales y recolección de residuos.

En este tipo de escalas, es común diseñar frases en sentido proactivo, es decir tomando medidas ambientales que la mayoría consideraría positivas, por tanto estar muy de acuerdo significaría aceptarlas. Otras en cambio, están diseñadas en sentido reactivo, donde estar muy de acuerdo significaría que acepta medidas que ante la mayoría de personas se considerarían negativas para el ambiente.

Si bien el método tradicional para medir el constructo de actitudes son las escalas Likert, hoy día se evalúan nuevas métodos basados en cuestionarios de preguntas abiertas y semiabiertas en las que se tiene la posibilidad de explorar las ideas de los estudiantes (Caurín y Gil, 2011). Estos cuestionarios resultan interesantes porque, en primera instancia, no sesgan la respuesta de los participantes, y además, permiten que la persona responda con criterios propios.

\section{MATERIALES Y MÉTODO}

La investigación se realizó siguiendo los lineamientos de la investigación acción en tanto que toma como punto de partida las nociones de los participantes y no investiga al azar. Además, de acuerdo con Vargas y Estupiñan (2012), insta a la comunidad a la acción reflexiva con miras a la construcción de una realidad mediada por el cambio de actitudes, las cuales se potencian cuando los problemas objeto de estudio se refieren a situaciones concretas que están inmersas en la realidad social, privilegiando la experiencia cotidiana.

Este tipo de investigación es más que pertinente en los procesos de EA porque todo proceso de transformación e innovación como el que propone el proyecto de investigación, debe ir acompañado en palabras de Mayer (1998) de una reflexión sobre la acción, sobre lo que se está haciendo y sobre lo que se va a hacer, a fin de no imponer actitudes socialmente aceptadas sino de aprender conjuntamente. De esta manera, la investigación acción es una metodología óptima para la exploración de problemas y potencialidades ambientales al ser un ejemplo de coherencia entre los métodos de reflexión que usa y la complejidad misma que encierran las dimensiones del ambiente.

\section{Participantes}

El instrumento de diagnóstico de actitudes hacia el agua, fue aplicado en el año 2012 como parte del proyecto "Agua Viva, un legado de nuestra cultura Muisca", orientado a desarrollar una estrategia didáctica para consolidar actitudes de compromiso, conocimiento y conservación hacia el agua. Se aplicó a un grupo de 33 estudiantes de grado séptimo de la Institución Educativa 
Francisco de Paula Santander del municipio de Ventaquemada, aledaño al Páramo de Rabanal (Boyacá).

El número total de participantes fue obtenido por conveniencia y facilidad de acceso a ellos. Además, para evitar falseamientos en las respuestas se evitó colocar nombres y apellidos y para ello se asignó un código que permite sistematizar mejor la información respetando la identidad del participante.

\section{Procedimiento}

Para la fase de exploración de las actitudes iniciales, que concierne a este artículo, y previo a implementar un proceso de acción que involucre cambios en patrones actitudinales, se estructuró una metodología en dos momentos: un primer momento de reconocimiento de la zona y los actores participantes, y un segundo momento de documentación y estructuración del diagnóstico. Se diseñó un cuestionario que permitió valorar las dimensiones conceptual, procedimental y axiológica del constructo actitud hacia el agua. Para ello se diseñó un libro código que en el caso de la dimensión conceptual contiene temas, cuestiones (subtemas), frases (ítems) e intencionalidad de las preguntas o enunciados propuestos en el cuestionario. Además, cada pregunta está diseñada teniendo en cuenta tres competencias definidas para las Ciencias Naturales, a saber: identificar, indagar y explicar.

Para proveer de validez el instrumento de diagnóstico, se sometió al método de validación por expertos, que resulta práctico para entender desde el sujeto la comprensión de la escala misma, teniendo en cuenta que los aportes que los validadores dan no se pueden extractar con un análisis factorial. Producto de las consideraciones y aportes de cada experto, se consolidó una primera sección con 12 preguntas de selección múltiple con única respuesta que valoraba la dimensión conceptual, y una segunda sección que planteaba 8 situaciones dilemáticas con opción de respuesta abierta y semiabierta para evaluar las dimensiones procedimental y afectiva. El hecho de encontrar este tipo de situaciones que implican toma de decisiones, repercute positivamente en la valoración de actitudes en tanto que en la medida en que se responda abiertamente a la pregunta, de acuerdo con Caurín (citado por Hernández, 2010), aumenta la posibilidad de inferir rasgos de la actitud no observables directamente. Además, se realizó un pilotaje con estudiantes de características similares al grupo participante y que está inmerso en la misma realidad contextual.

\section{Procesamiento y análisis de la información}

Posterior a la aplicación de los instrumentos a la población participante, se realizó un análisis de las respuestas. Para el análisis de la primera sección se establecieron variables, sus características y se aplicaron estadísticos descriptivos mediante el software estadístico SPSS versión 17, agrupando las respuestas obtenidas en niveles de competencia según la Tabla 1. 
Tabla 1. Niveles de competencia establecidos para evaluar la dimensión conceptual del constructo actitud hacia el agua

\begin{tabular}{|l|l|}
\hline NIVEL DE COMPETENCIA & DESCRIPCION \\
\hline Nivel A & $\begin{array}{l}\text { Denota el nivel básico de complejidad. Los niños y niñas } \\
\text { aportan explicaciones desde su experiencia cotidiana. } \\
\text { Establece relaciones sencillas entre fenómenos. }\end{array}$ \\
\hline Nivel B & $\begin{array}{l}\text { Nivel intermedio. Los niños y niñas reconocen y analizan } \\
\text { fenómenos haciendo uso de teorias. Además, las } \\
\text { explicaciones que aportan a los fenómenos expuestos son } \\
\text { más elaboradas. }\end{array}$ \\
\hline Nivel C & $\begin{array}{l}\text { Los niños y niñas realizan inferencias con base en la } \\
\text { comprensión de fenómenos a la luz de teorías y conceptos } \\
\text { universales, lo que implica un mayor grado de abstracción. }\end{array}$ \\
\hline
\end{tabular}

Posterior a ello se realizó un análisis con el test no paramétrico $\mathrm{X} 2$, a fin de establecer diferencias significativas entre las respuestas de los estudiantes participantes. Para el análisis del segundo instrumento se establecieron 6 categorías de análisis determinadas a través de un proceso deductivo que, en palabras de Pantoja (2009), fueron creadas atendiendo a las necesidades y potencialidades de la investigación, y se diferencian claramente en la medida en que valoran aspectos distintos de la actitud hacia el agua. Se procedió a hacer un análisis cualitativo haciendo uso del software Atlas.ti, consolidando a partir de los saberes reales de los estudiantes, categorías inductivas que fueron analizadas a la luz de diversos autores expertos en el tema. Para el análisis de datos cualitativos se llevó a cabo una secuenciación de los mismos, partiendo de la toma de los datos, y siguiendo tres niveles de análisis: textual, donde se realizó una reducción inicial de texto a fin de obtener los apartes más relevantes para la investigación; nivel conceptual, en el que se establecieron categorías y subcategorías inductivas con base en los saberes reales de los participantes; y un nivel interpretación, en el que se realizó una triangulación hermenéutica de los datos a la luz de las categorías. Este esquema no fue de tipo secuencial, sino más bien circular y siempre hubo la necesidad de volver y retomar el nivel textual (Pantoja, 2009).

\section{RESULTADOS}

\section{Resultados dimensión conceptual}

Entre los estudiantes participantes, con dos grados de libertad y un nivel de confianza del $95 \%$ se encontraron diferencias estadísticamente significativas en todas las preguntas del cuestionario excepto en las número 4 y 7 (Tabla 2). 
Tabla 2. Resultados cuestionario dimensión conceptual

\begin{tabular}{|c|c|c|c|c|c|c|c|c|}
\hline \multirow{2}{*}{$\begin{array}{l}\text { Variable } \\
\text { Name }\end{array}$} & \multirow[b]{2}{*}{ Definition } & \multirow[b]{2}{*}{ Values } & \multirow{2}{*}{$\begin{array}{c}\text { Frequency } \\
\text { (Observed N) }\end{array}$} & \multirow{2}{*}{$\begin{array}{c}\text { Valid } \\
\text { Percent }\end{array}$} & \multirow{2}{*}{$\underset{\mathrm{N}}{\text { Expected }}$} & \multirow[b]{2}{*}{ Residual } & \multicolumn{2}{|c|}{ Test Statistics } \\
\hline & & & & & & & $\begin{array}{l}\text { Chi- } \\
\text { Square }\end{array}$ & $\begin{array}{l}\text { Asymp. } \\
\text { Sig. }\end{array}$ \\
\hline \multirow{4}{*}{$\underset{1}{\text { Pregunts }}$} & \multirow[b]{3}{*}{$\begin{array}{c}\text { Estructura química } \\
\text { del agus }\end{array}$} & Nivela & 1 & 3,0 & 11,0 & $-10,0$ & \multirow[t]{4}{*}{252738} & \multirow[t]{4}{*}{.000} \\
\hline & & Nivels & 8 & 24,2 & 11,0 & $-3,0$ & & \\
\hline & & Nivelc & 24 & 72,7 & 11,0 & 13,0 & & \\
\hline & Total & & 33 & 100,0 & & & & \\
\hline \multirow{4}{*}{$\begin{array}{l}\text { Pregunts } \\
2\end{array}$} & \multirow[b]{3}{*}{$\begin{array}{l}\text { Enlaces propios de } \\
\text { ls moléculs de sgus }\end{array}$} & NivelA & 3 & 9,1 & 11,0 & $-8,0$ & \multirow[t]{4}{*}{9.2559} & \multirow[t]{4}{*}{.009} \\
\hline & & NivelB & 17 & 51,5 & 11,0 & 5,0 & & \\
\hline & & Nivelc & 13 & 39,4 & 11,0 & 2,0 & & \\
\hline & Total & & 33 & 100,0 & & & & \\
\hline \multirow{4}{*}{$\begin{array}{l}\text { Pregunts } \\
3\end{array}$} & \multirow{3}{*}{$\begin{array}{l}\text { Fenómenos de } \\
\text { cohesióny adhesión }\end{array}$} & NivelA & 5 & 18,2 & 11,0 & $-5,0$ & \multirow[t]{4}{*}{27.4559} & \multirow[t]{4}{*}{.000} \\
\hline & & Nivels & 2 & 6,1 & 11,0 & $-9,0$ & & \\
\hline & & Nivelc & 25 & 75,8 & 11,0 & 14,0 & & \\
\hline & Total & & 33 & 100,0 & & & & \\
\hline & & NivelA & 15 & 45,5 & 11,0 & 4,0 & 2.1823 & .336 \\
\hline Pregunts & & Nivel B & 9 & 27,3 & 11,0 & $-2,0$ & & \\
\hline 4 & $\begin{array}{c}\text { Calorespecifico del } \\
\text { ggus }\end{array}$ & Nivelc & 9 & 27,3 & 11,0 & $-2,0$ & & \\
\hline & Total & & 33 & 100,0 & & & & \\
\hline & & Nivela & 13 & 39,4 & 11,0 & 2,0 & 70913 & .029 \\
\hline Pregunts & & Nivel B & 4 & 12,1 & 11,0 & -7.0 & & \\
\hline 5 & $\begin{array}{c}\text { Fases del ciclo del } \\
\text { ggus }\end{array}$ & Nivelc & 16 & 48,5 & 11,0 & 5,0 & & \\
\hline & Total & & 33 & 100,0 & & & & \\
\hline & & NivelA & 3 & 9,1 & 11,0 & $-8,0$ & 39.4559 & .000 \\
\hline Pregunts & Rios en el & Nivels & 2 & 6,1 & 11,0 & $-9,0$ & & \\
\hline 6 & $\begin{array}{c}\text { departsmento de } \\
\text { Boyscá }\end{array}$ & Nivelc & 28 & 84,8 & 11,0 & 17,0 & & \\
\hline & Total & & 33 & 100,0 & & & & \\
\hline & & Nivela & 8 & 24,2 & 11,0 & $-3,0$ & 12736 & .529 \\
\hline Prequnts & & Nivels & 13 & 39,4 & 11,0 & 2,0 & & \\
\hline- & $\begin{array}{c}\text { Función de los } \\
\text { páramos }\end{array}$ & Nivelc & 12 & 36,4 & 11,0 & 1,0 & & \\
\hline & Total & & 33 & 100,0 & & & & \\
\hline & & NivelA & 3 & 9,1 & 11,0 & $-8,0$ & 20.3543 & .000 \\
\hline & & NivelB & 7 & 21,2 & 11,0 & $-4,0$ & & \\
\hline 8 & $\begin{array}{c}\text { antropicas hacis el } \\
\text { pársmo }\end{array}$ & Nivelc & 23 & 69,7 & 11,0 & 12,0 & & \\
\hline & Total & & 33 & 100,0 & & & & \\
\hline & & NivelA & 25 & 75,8 & 11,0 & 14,0 & 27.5553 & .000 \\
\hline & Importancis & Nivel B & 2 & 6,1 & 11,0 & $-9,0$ & & \\
\hline $\log _{9}$ & $\begin{array}{c}\text { biológics de los } \\
\text { páramos }\end{array}$ & Nivelc & 6 & 18,2 & 11,0 & $-5,0$ & & \\
\hline & Total & & 33 & 100,0 & & & & \\
\hline & El mito como & NivelA & 9 & 27,3 & 11,0 & $-2,0$ & 9.4559 & .009 \\
\hline Pregunts & estrategis de & Nivels & 5 & 15,2 & 11,0 & $-6,0$ & & \\
\hline 10 & $\begin{array}{c}\text { protección del sgus } \\
\text { y las lagungs }\end{array}$ & Nivelc & 19 & 57,5 & 11,0 & 8,0 & & \\
\hline & Total & & 33 & 100,0 & & & & \\
\hline & & NivelA & 8 & 24,2 & 11,0 & $-3,0$ & 272738 & .000 \\
\hline Pregunts & El mito de origen y & Nivel B & 2 & 6,1 & 11,0 & $-9,0$ & & \\
\hline 11 & $\begin{array}{c}\text { su relación con el } \\
\text { sgus }\end{array}$ & Nivelc & 23 & 69,7 & 11,0 & 12,0 & & \\
\hline & Total & & 33 & 100,0 & & & & \\
\hline & & NivelA & 17 & 51,5 & 11,0 & 5,0 & 6.5459 & .038 \\
\hline & & Nivels & 5 & 15,2 & 11,0 & $-6,0$ & & \\
\hline 12 & $\begin{array}{l}\text { Sacra lidad alrededor } \\
\text { de las lagungs }\end{array}$ & Nivelc & 11 & 33,3 & 11,0 & .0 & & \\
\hline & Total & & 33 & 100,0 & & & & \\
\hline
\end{tabular}

En la pregunta 1, estructura de la molécula del agua, el 73\% de los estudiantes comprenden claramente que la imagen que se representa en el cuestionario corresponde a la molécula de agua y que está formada químicamente por dos átomos de hidrógeno y uno de oxígeno. Las diferencias estadísticamente significativas radican en que un $24 \%$ de los estudiantes si bien reconocen la molécula de agua, creen que el átomo de oxígeno de mayor 
tamaño atrae a los átomos de hidrógeno formándose enlaces iónicos. Apenas un 3\% de los estudiantes no definen el agua como una molécula sino más bien como un elemento puro, de ahí su transparencia.

En la pregunta 2, referida a los enlaces propios de la molécula del agua, el $52 \%$ de los niños y niñas creen que el oxígeno y el hidrógeno están unidos mediante un enlace iónico, lo anterior debido quizá a que tienen la concepción de que el átomo de oxígeno de mayor tamaño atrae a los átomos de hidrógeno, cayendo en un error conceptual. Sin embargo, el 39\% de los estudiantes deducen que el enlace que une dichos átomos es de tipo covalente, lo que los sitúa en un nivel $\mathrm{C}$ de competencia.

En la pregunta 3, el agua desde el punto de vista físico, se establecen diferencias estadísticamente significativas teniendo en cuenta que el $76 \%$ de los niños y niñas asocian el transporte de agua de la raíz a las hojas a los fenómenos de adhesión y cohesión de las moléculas de agua, lo que los sitúa en un nivel C de competencia. Caso contrario con el $18 \%$ de los estudiantes que afirman que el agua es liviana y de ahí que sea fácil su transporte. Por último, el $6 \%$ de los estudiantes afirman que es gracias a los enlaces covalentes entre moléculas de agua que se forman escaleras permitiendo ascender el agua hasta las hojas.

Al cuestionarles sobre el ciclo del agua, ocurre un caso similar tras mostrar un esquema que lo resume. El $49 \%$ de los estudiantes concluyen que las nubes se forman por evaporación del agua líquida, situándolos en un nivel $\mathrm{C}$ de competencia. En contraste, un $39 \%$ de los niños y niñas consideran que las nubes siguen todo el recorrido de las flechas del ciclo esquematizado acudiendo únicamente a una interpretación básica, lo que los sitúa en un nivel A de competencia. Por último, un $12 \%$ de los participantes acuden a sus vivencias cotidianas y afirman que en el ciclo del agua las nubes producen agua en forma de lluvia.

Respecto a la pregunta de la disponibilidad y oferta hídrica en el departamento de Boyacá, el $85 \%$ de los estudiantes se agrupan en un nivel $C$ de competencia teniendo en cuenta que comprenden que en Boyacá existe gran riqueza hídrica y que a pesar de que el agua es considerada recurso renovable, es responsabilidad de todos cuidarla porque esta no es inagotable. La diferencia estadísticamente significativa radica en que el $9 \%$ de los niños y niñas consideran, al observar el mapa de Boyacá con sus ríos y cuencas, que en este departamento el agua es abundante y por ende inagotable, de ahí que no se procuren acciones a favor de su conservación.

En la pregunta 8 se establecían las principales amenazas antrópicas al páramo y se inducia a los niños y niñas a determinar las implicaciones que dichas amenazas representan para las especies endémicas de los ecosistemas de alta montaña. A lo cual, el 70\% afirmaron que las consecuencias serían fatales porque al ser especies endémicas solo prosperan en el páramo. El $21 \%$ de los estudiantes reconocen que sería una situación preocupante, sin embargo el término endémico les 
sugiere que pueden prosperar en otro lugar. Aún más desconcertante resulta el $9 \%$ de los estudiantes que creen que gracias a las quemas y contaminación del hombre en los páramos las especies se adaptan y, por ende, no es motivo de preocupación que se sigan realizando indiscriminadamente dichas actividades.

Cuando se les cuestionó a los niños y niñas sobre la importancia biológica de los páramos en términos de supervivencia de los seres humanos, el $76 \%$ de los participantes asumen que dicha importancia radica en que los páramos son hábitat de especies de plantas y animales, si bien esta es una función sumamente importante, lo es aún más el hecho de que los páramos sean los lugares que surten de agua a las fuentes hídricas, respuesta que aseveraron apenas un $18 \%$ de los estudiantes. Mientras que el $6 \%$ reconocen que son lugares llenos de cultura y tradición, lo que representa mayor importancia sobre el abastecimiento de agua.

En la pregunta 10 se les proporcionó a los niños y niñas una imagen donde se personifican algunas deidades de la cultura Muisca, y a continuación se les cuestionó sobre cómo se debían concebir esos imaginarios de mito dentro de la generación actual. El 58\% de los estudiantes afirman que el imaginario de mito del Pueblo Muisca debe ser considerado como una estrategia de protección a los páramos. En contraste, un 27\% de los niños y niñas concibieron el mito como ilusorio y primitivo, desconociendo que al darle animismo a elementos como las montañas y el agua, los Muiscas mantenían un equilibrio ecológico en el ambiente. El $15 \%$ de los participantes reconocieron que aunque los mitos son creaciones fantasiosas y carentes de realidad, en este caso son patrimonio de la sabiduría del Pueblo Muisca.

En la pregunta 11 , el $70 \%$ de los estudiantes afirman que tras la lectura del mito de Origen de Bachué, el elemento fundamental a través del cual gira la historia es el agua, reconociendo así que para los Muiscas el agua era fuente de vida literalmente, de ahí que Bachué su progenitora haya salido del agua y una vez formado el Pueblo Muisca, volvió a ella. Sin embargo, un 24\% de los estudiantes se situaron en un nivel $A$ de competencia al afirmar que el centro de la historia son los seres humanos representado en el Pueblo de Iguaque que formó Bachué, demostrándose una clara tendencia antropocentrista alrededor de los elementos naturales que dieron origen a la creación.

En la pregunta 12, se cuestionaba acerca de la concepción de sacralidad que tenían lugares como los páramos y las lagunas para los Muiscas, en razón a ello, el 52\% de los niños y niñas se situaron en nivel A de competencia al afirmar que los páramos y las lagunas eran sitios de fiesta, romerías y adoración, desconociendo pese a la lectura previa, que los Muiscas no osaban cortar ni una rama de esos sitios y solo ascendían a los páramos en busca de medicinas cuando realmente era necesario, respuesta con la que estuvo de acuerdo un 33\% de los participantes. El 15\% afirmaron que los páramos no eran 
espacios sacros, sino lugares de resguardo tras la llegada de la conquista española.

\section{Resultados dimensión operacional y afectiva del constructo actitud hacia el agua}

En este punto la información se interpretó en la medida en que fue generada por los niveles de análisis atendiendo a las 6 categorías deductivas de análisis. Es pertinente mencionar que los resultados que se presentan constan de dos partes: la primera, saberes que reflejan actitudes, compila algunas de las respuestas literales de los niños; y la segunda, interpretación, comprende las categorías inductivas que se extractaron producto de la lectura y el análisis textual de las respuestas de los niños y niñas.

\section{Categoría: Situación y problemáticas asociadas al agua}

Producto de las respuestas de los niños y niñas se establecen 3 categorías inductivas que resumen las causas generales posibles que engloban las situaciones y problemáticas relacionadas con el agua, a saber: causas antrópicas, causas naturales, y gestión y responsabilidad comunitaria. Apartes de las respuestas de los participantes se muestran en la Tabla 3.

Tabla 3. Datos obtenidos a partir de la sistematización de los resultados pregunta 1 a la luz de la categoría deductiva 'Situación y problemáticas asociadas al agua'

\begin{tabular}{|c|c|c|}
\hline \multicolumn{3}{|l|}{ CODIGO PREGUNTA:AS.BI. } \\
\hline \multirow[b]{2}{*}{ SABERES QUE REFLEJAN ACTITUDES } & \multicolumn{2}{|c|}{ INTERPRETACION } \\
\hline & SUBCATEGORIASINDUCTNAS & $\begin{array}{l}\text { CATEGORIAS } \\
\text { INDUCTIVAS }\end{array}$ \\
\hline $\begin{array}{l}\text { Porque el hombre ha contaminado la maycr cartidad de agua arrojando basura u } \\
\text { otros desechos que no siven" (1F1, 1F2, 1F7, 1F8, 1F10, 1F18, 1M19, 1F20. } \\
\text { 1M21, 1F26, 1F28, 1M32) }\end{array}$ & Contaminacion & \multirow{8}{*}{ Causas Antrópicas } \\
\hline $\begin{array}{l}\text { Las personas gascan las reservas sin pensar que les pueden hacer fots (IF } 2 \text {. } \\
1 F 5,1 F 6,1 F 9,1 \mathrm{M} 12,1 \mathrm{~F} 20,1 \mathrm{~F} 23,1 \mathrm{M} 32)\end{array}$ & Despifarro & \\
\hline 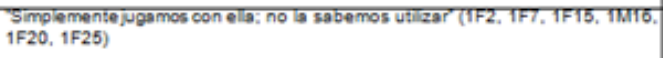 & Mal uso & \\
\hline $\begin{array}{l}\text { Porque cuando hubo mucha no fuimos conscentes que nos liba a ser tata } \\
\text { despues:". (1F11) }\end{array}$ & Fatta de conclencia & \\
\hline 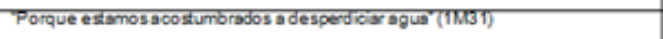 & Pिएका०ड & \\
\hline Porque hay mucha gerte" & Sobrepoblación & \\
\hline Por el ecosistema que esta muy acabsdo.... (1/727) & Deterioco del ecosistema & \\
\hline 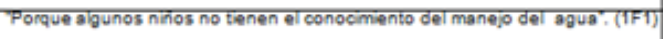 & Desconocimento & \\
\hline $\begin{array}{l}\text { Esta stuación se debe a que hace mucho sol y entonces los nios, se secan" } \\
(1 F 1,1 F 2,1 M 12,1 M 17,1 M 19,1 F 23,1 M 24,1 F 28,1 F 29,1 F 33)\end{array}$ & Variaciones climáticas & \multirow[t]{2}{*}{ Causas Naturales } \\
\hline Porque cuando se evapora y se secan los lagos y los rios (t/uch) & Evaporaoon & \\
\hline "Las camparias no dan buenos resulados ni generan impacto" (1F 11) & 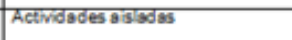 & \multirow{4}{*}{$\begin{array}{l}\text { Gestión y } \\
\text { responsabilidad } \\
\text { comunitaris }\end{array}$} \\
\hline Es portalar arboles (1F25. 1M130) & Tola y fita de siembra de siboles & \\
\hline Porque no hay planes para las épocas de sequía"(1F9. 1M16) & Falta de sistemas de prevención & \\
\hline 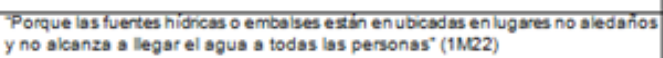 & Ubicación noestratéges & \\
\hline
\end{tabular}




\section{Categoría: Alternativas de conservación del agua}

En esta categoría se pretendió indagar sobre la posición de los participantes respecto a la aplicación de tecnologías limpias y la participación de las comunidades en procesos de Educación Ambiental como estrategias de conservación y manejo del agua. La Tabla 4 sintetiza las respuestas obtenidas.

Tabla 4. Datos obtenidos a partir de la sistematización de los resultados pregunta 2 a la luz de la categoría deductiva 'Alternativas de conservación del agua'

\begin{tabular}{|c|c|c|}
\hline \multicolumn{3}{|l|}{ CODIOO PRECUNTAE AS.B2. } \\
\hline \multirow[b]{2}{*}{ SMBERES QUE REFLEJAN ACTTUDES } & \multicolumn{2}{|c|}{ INTERPFETACION } \\
\hline & SUBCATEGORIASINOUCTNAS & $\begin{array}{l}\text { CATEGOAIAS } \\
\text { INOUCTIVAS }\end{array}$ \\
\hline 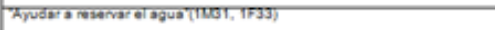 & 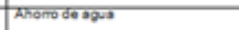 & \multirow{8}{*}{$\begin{array}{l}\text { Aremisvas de } \\
\text { conservacion }\end{array}$} \\
\hline 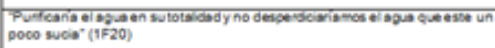 & Aprovechamienso & \\
\hline 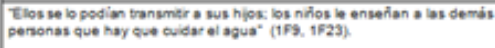 & Conocimierto Tansusenersiond & \\
\hline 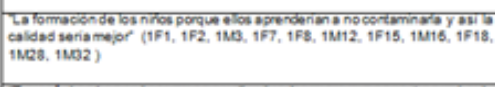 & Edvesosion & \\
\hline 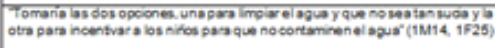 & Educación-Tenologis & \\
\hline 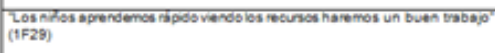 & Inmerión envel contedo & \\
\hline 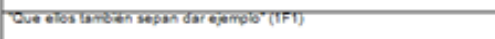 & 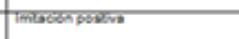 & \\
\hline 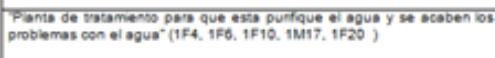 & Solvoines twenoligicas & \\
\hline 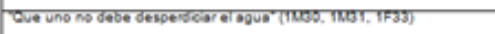 & 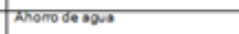 & \multirow{5}{*}{ imooctos } \\
\hline 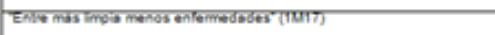 & sacs & \\
\hline 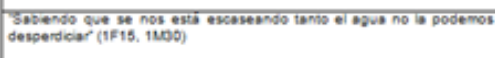 & Concensos & \\
\hline 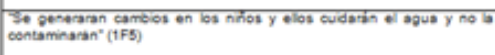 & Cuidado & \\
\hline 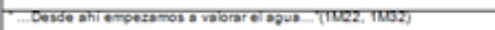 & tracason & \\
\hline
\end{tabular}

\section{Categoría: Toma de decisiones frente al aprovechamiento del agua}

Esta categoría indagó respecto a las actitudes de los estudiantes frente a la producción y consumo de productos modernos entendiendo que son responsables, en gran medida, de la contaminación de las fuentes hídricas y despilfarro de agua. Producto del análisis textual y conceptual de las respuestas de los niños se establecieron 2 categorías inductivas de análisis (Tabla 5). 
Tabla 5. Datos obtenidos a partir de la sistematización de los resultados pregunta 3 a la luz de la categoría deductiva 'Toma de decisiones frente al aprovechamiento del agua'

\begin{tabular}{|c|c|c|}
\hline \multicolumn{3}{|l|}{ CODIGO PREGUNTACAS. 83.} \\
\hline \multirow[b]{2}{*}{ SABERES QUE REFLEJAN ACTITUDES } & \multicolumn{2}{|c|}{ INTERPFETACION } \\
\hline & SUBCATEGORIASINDUCTNAS & $\begin{array}{l}\text { CATEGOFIAS } \\
\text { INDUCTIVAS }\end{array}$ \\
\hline 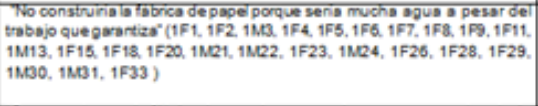 & $\begin{array}{l}\text { Profizacion del agua frertes is } \\
\text { industra }\end{array}$ & \multirow{3}{*}{$\begin{array}{l}\text { Acttud frente al } \\
\text { aprovechamiento } \\
\text { del agus }\end{array}$} \\
\hline 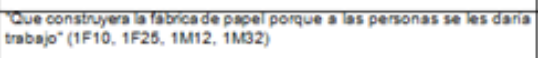 & $\begin{array}{l}\text { Pronzzacón dela industria frented } \\
\text { apus }\end{array}$ & \\
\hline 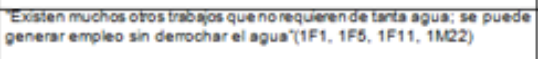 & 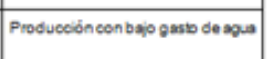 & \\
\hline Que no la constuya porque el pueblo se podna contaminar mas" (IFA) & Aumemiode consminsoon & \multirow[b]{2}{*}{ Consecuencias } \\
\hline 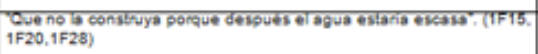 & Evasez deagua & \\
\hline
\end{tabular}

\section{Categoría: Actores responsables frente al agua}

Se propuso un enunciado en que se ofrecían cifras del gasto de agua en las zonas urbanas y en el sector agrícola que representa una de las principales actividades económicas de los pobladores de zonas rurales. A propósito se indagó sobre la percepción de los niños y niñas frente a las responsabilidades de los actores sociales respecto al agua. La Tabla 6 sintetiza los principales hallazgos.

Tabla 6. Datos obtenidos a partir de la sistematización de los resultados pregunta 4 a la luz de la categoría deductiva 'Actores responsables frente al agua'

\begin{tabular}{|c|c|c|}
\hline \multicolumn{3}{|l|}{ CODIGO PREGUNTA:ASCI. } \\
\hline \multirow[b]{2}{*}{ SABERES QUE REFLEJAN ACTITUDES } & \multicolumn{2}{|c|}{ INTERPRETACIÓN } \\
\hline & SUBCATEGORIASINDUCTNAS & $\begin{array}{l}\text { CATEGORIAS } \\
\text { INDUCTIVAS }\end{array}$ \\
\hline 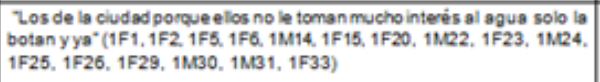 & Habitantes de la ciudad & \multirow[b]{3}{*}{$\begin{array}{l}\text { Actores } \\
\text { responsables }\end{array}$} \\
\hline 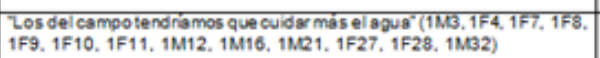 & Habtantes del campo & \\
\hline $\begin{array}{l}\text { Yo dría que los dos porque tarto en el campo como en la civdad gastan } \\
\text { agua": (1M13, 1M17, 1F18, 1M19) }\end{array}$ & Todos & \\
\hline $\begin{array}{l}\text { Los de la oudad porque saben que sino la cuidan no les van a dar más } \\
\text { flotas de agua" (1F6, } 1 F 29,1 \mathrm{M} 30 \text { ) }\end{array}$ & Escasez del agua & \multirow{3}{*}{$\begin{array}{l}\text { Razones de } \\
\text { responsabilidac } \\
\text { oivdadanos }\end{array}$} \\
\hline Los de la ciudad porque elos is contaminan" (1MT4) & Contaminacisn & \\
\hline $\begin{array}{l}\text { Los de la oudad porque elos el agua que utilizan muchas veces la } \\
\text { desperdioian". (1F1, 1F2,1M17, 1F20, 1M22) }\end{array}$ & Despifarto & \\
\hline Los del campo benen mas agua y vegetacon" (1F6, 1F28) & Abundancia & \multirow{3}{*}{$\begin{array}{l}\text { Razones de } \\
\text { responsabilidad } \\
\text { campo }\end{array}$} \\
\hline 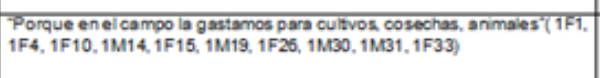 & $\begin{array}{l}\text { Mantenimient de Cutivos y } \\
\text { actividades pastorles }\end{array}$ & \\
\hline $\begin{array}{l}\text { Zos del campo porque siembran con pesticidas y produdos químicos los } \\
\text { cuales necestan mucha agua":(1F9.1MM2) }\end{array}$ & Sustancias cortaminartes & \\
\hline Tanto en el campo como en la cudad pastan agua. (1F18) & Gasto & $\begin{array}{l}\text { Razones de } \\
\text { responsabilidad } \\
\text { conjunts }\end{array}$ \\
\hline
\end{tabular}

\section{Categoría: Agua como elemento esencial}

Se expuso una situación dilemática en la cual se planteaba la posibilidad de construir una carretera que generaría grandes ingresos económicos pero que implicaría la desecación de un 
pozo de agua. En la Tabla 7 se resumen las actitudes de los participantes frente al agua y al dinero como factores necesarios en las actividades humanas.

Tabla 7. Datos obtenidos a partir de la sistematización de los resultados pregunta 5 a la luz de la categoría 'Agua como elemento esencial'

\begin{tabular}{|c|c|c|}
\hline \multicolumn{3}{|l|}{ CODIGO PREGUNIA: VAEL } \\
\hline \multirow[b]{2}{*}{ SABERES QUE REFLEJAN ACTITUDES } & \multicolumn{2}{|c|}{ INTERPRETACION } \\
\hline & SUBCATEGORIASINDUCTIVAS & $\begin{array}{l}\text { CATEGORIAS } \\
\text { INDUCTIVAS }\end{array}$ \\
\hline $\begin{array}{l}\text { Igual para bañarnos tocania ir hasta el pozo mayor; porque no habria } \\
\text { alimentos en el pueblo y la gente moriria de hambre". (1F 10, 1F } 33 \text { ) }\end{array}$ & Serviciohumano & \multirow{7}{*}{$\begin{array}{c}\text { Razones para } \\
\text { privilegiar el agua }\end{array}$} \\
\hline "Sin agua mis cultivos se dañarian". (1/F2, 1M24, 1Ma12) & Evita daño a cultivos & \\
\hline 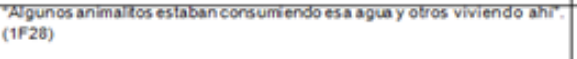 & Hibitat de especies & \\
\hline 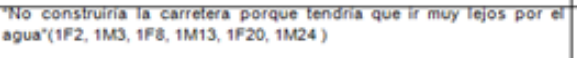 & Lejania de is fuente hidnica & \\
\hline $\begin{array}{l}\text { "No porque no podriair por agua a su pozo y jamas podria tener mas agua } \\
\text { de la que tenia" (1M90) }\end{array}$ & Limitado & \\
\hline $\begin{array}{l}\text { "No la construira porque las plata que le por da la verta de los productos la } \\
\text { gasta en combustible para ir agua al pozo". (1M16) }\end{array}$ & Mayor gasto de dinero & \\
\hline 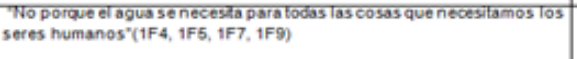 & Necessidad & \\
\hline $\begin{array}{l}\text { Yo construiria la carreters porque los alimentos se le quedarian en las } \\
\text { finca" (1F10, 1F 18, 1F29) }\end{array}$ & Agricultura & \multirow{3}{*}{$\begin{array}{c}\text { Condiciones que } \\
\text { priman sobre ell } \\
\text { agua }\end{array}$} \\
\hline 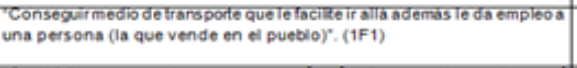 & Comodidad & \\
\hline 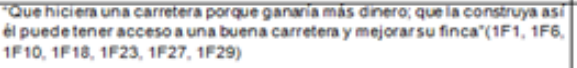 & Ingres os económicos & \\
\hline
\end{tabular}

\section{Categoría: Agua como elemento de identidad}

Bajo las pretensiones de identificar las actitudes de los estudiantes hacia el agua entendida desde su complejidad ambiental, se planteó que el suministro de agua para una comunidad aislada implicaba generar daño al pasar un tubo por un bosque y afectar una laguna sagrada para una cultura indígena ancestral, las opiniones se dividieron fuertemente según los resultados de la Tabla 8.

Tabla 8. Datos obtenidos a partir de la sistematización de los resultados pregunta 6 a la luz de la categoría 'Agua como elemento de identidad'

\begin{tabular}{|c|c|c|}
\hline \multirow[b]{2}{*}{ SABERES QUE REFLESAN ACTITUDES } & \multicolumn{2}{|c|}{ INTERPRETACION } \\
\hline & SUBCATEGORLASINDUCTIVAS & $\begin{array}{l}\text { CATEGOAISS } \\
\text { INDUCTIVAS }\end{array}$ \\
\hline 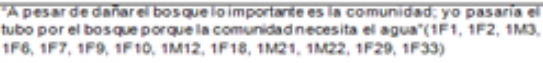 & Antropocertismo ded agua & \multirow{3}{*}{$\begin{array}{l}\text { Agua y su } \\
\text { compleejdad } \\
\text { ambiental }\end{array}$} \\
\hline 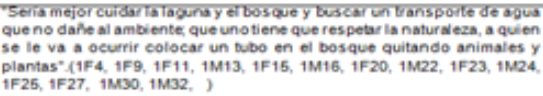 & $\begin{array}{l}\text { Respeto a los componertes } \\
\text { naturales y culturales }\end{array}$ & \\
\hline $\begin{array}{l}\text { "Mejor noirrespetaria laguna de los indoenas, porque puede tener algo" } \\
(1 F 9,1 M 14,1 M 19,1 F 26 \text { ) }\end{array}$ & Sistema de creendas & \\
\hline 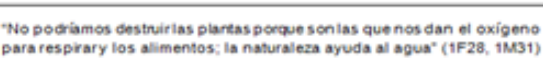 & Relaciones de interdependenos & $\begin{array}{l}\text { Cacsas deros } \\
\text { patrones de } \\
\text { actuación }\end{array}$ \\
\hline 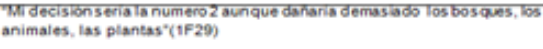 & Concienola del daño & \multirow{2}{*}{$\begin{array}{l}\text { Conseovencias de } \\
\text { los patrones de } \\
\text { actuación }\end{array}$} \\
\hline 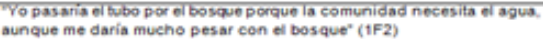 & Sentimientes de culpa & \\
\hline
\end{tabular}




\section{Categoría: Percepción afectiva del agua}

En la Tabla 9 se observan las percepciones en términos de definición, importancia, origen y usos y prácticas alrededor del agua.

Tabla 9. Datos obtenidos a partir de la sistematización de los resultados pregunta 7 a la luz de la categoría 'Percepción afectiva del agua'

\begin{tabular}{|c|c|c|}
\hline \multirow[b]{2}{*}{ SABERES QUE REFLEIAN ACTITUDES } & \multicolumn{2}{|c|}{ MTERPRETACION } \\
\hline & SUBCATEGORLASINOUCTNAS & $\begin{array}{l}\text { CATEGORIKS } \\
\text { INDUCTNAS }\end{array}$ \\
\hline 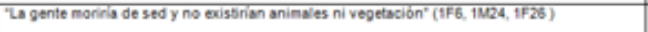 & Fuctor se supenwenda & \\
\hline "Podemos hase un uso adocuaso para el agua' (172h) & Uso humano & Imporanca del agua \\
\hline 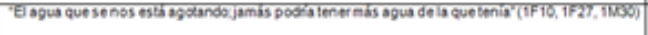 & Umitaso & Concepto \\
\hline 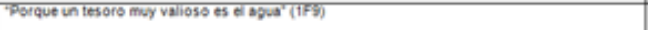 & Tesoro & \\
\hline 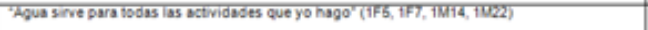 & semico & \\
\hline 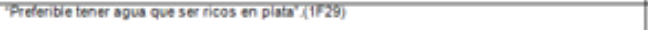 & Varor & \\
\hline 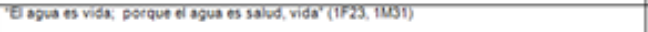 & Via & \\
\hline 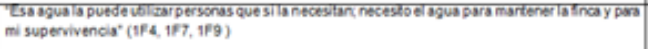 & Necesisas & \\
\hline "Epreciado lquido que es el agua', (1M-19) & Lquido preciado & \\
\hline 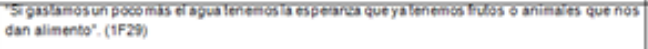 & Reemplazable & \\
\hline 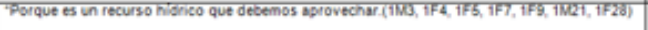 & Recorso & \\
\hline 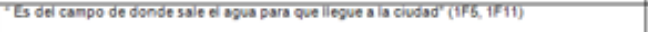 & $\operatorname{compo}$ & \\
\hline "Des nasesero or aguar. (IMT) & Nacederos & Origen \\
\hline 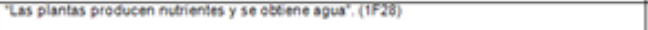 & De los nutriertes delas raicen & \\
\hline 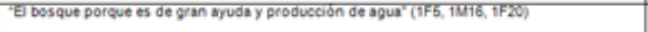 & Bosques & \\
\hline "Los arboles y estos son grandes tagricas de agus" (1M14) & Arsoles & \\
\hline
\end{tabular}

\section{DISCUSIÓN}

La dimensión conceptual del constructo actitud hacia el agua

Al analizar los resultados obtenidos en el conjunto de preguntas que daban cuenta de la dimensión conceptual, se observa que en todas las preguntas en que se establecieron diferencias estadísticamente significativas, el nivel C de competencia predominó. Esto implica que los participantes tienen conceptos claros en lo referente al agua desde el punto de vista químico y físico, es decir aplican teorías aprendidas y las transpolan para comprender que el agua es una molécula, que está unida por enlaces covalentes y que entre sus propiedades físicas figuran la cohesión y la adhesión. Caso similar ocurre con el agua desde el punto de vista ecológico. Se comprenden las fases que conforman el ciclo del agua, la riqueza hídrica en el departamento de Boyacá y la responsabilidad que ello implica, las consecuencias que traen para las especies endémicas actividades como quemas y extensión de cultivos en los ecosistemas de Páramo. Sin embargo, cuando se cuestiona sobre los sistemas socio-culturales alrededor del agua, las respuestas puntúan en niveles A y B de competencia. Así cuando se pregunta sobre la importancia de los páramos en 
términos de supervivencia para la especie humana, se reitera que se adjudica a las plantas y animales, desconociendo la función de los ecosistemas de alta montaña en la regulación hídrica; o cuando se cuestiona sobre el carácter sacro de las lagunas y los páramos para los Muiscas, simplemente se tergiversa la palabra adoración y se convierte en sinónimo de fiesta.

De lo anterior se extraen dos conclusiones: la primera es alentadora ya que al situar la mayoría de respuestas en nivel C de competencia, no solo se deduce un alto grado de abstracción entre los participantes, sino que se obtiene certeza en la posibilidad de modificar actitudes ambientales teniendo en cuenta que para adoptarlas es necesario tener un fuerte componente conceptual (Caurín y Gil, 2011), esto es: preconceptos claros, definidos y necesariamente positivos (Hernández, 2010). Lo anterior nos lleva a la segunda conclusión que suscita incongruencias, teniendo en cuenta que, si se parte del supuesto de que en las escuelas se están enseñando conceptos científicamente aceptados acerca del agua, entonces los problemas ambientales asociados a este líquido no radican en el conocimiento que los participantes tienen en relación a la situación objeto de estudio. Lo anterior concuerda con el trabajo de Mayer (1998), quien afirma que es una trampa pretender que la solución a los problemas ambientales está en el conocimiento objetivo de los mismos. Es necesario pensar entonces que las actuaciones de un individuo se mueven en otros campos además del conocimiento, campos que implican fuertes vínculos emocionales y como afirma Schibeci (citado por Caurín y Gil, 2011) no se trata entonces de valorar la actitud hacia el agua únicamente haciendo escalas que den cuenta del rendimiento escolar en sus dimensiones biológica, química y física a través de relaciones estadísticas. Es necesario atreverse a poner en un mismo plano los sentimientos y las propensiones a actuar según lo que se piensa y siente respecto al agua.

Análisis de las categorías inductivas atendiendo a las dimensiones procedimental y afectiva de las actitudes

En cuanto a la categoría Situación y problemáticas asociadas al agua, se establecen 3 categorías inductivas. En lo que respecta a las causas antrópicas, se rescatan varios aspectos: primero, el hecho de que en la mayoría de respuestas los participantes no se incluyen como agentes causantes de los problemas asociados al agua; esto se infiere cuando se habla de las personas en términos excluyentes. Segundo, la tendencia de resaltar los hábitos y el desconocimiento como causa de problemas relacionados con el agua, vislumbrando la pertinencia misma del ejercicio investigativo que se realiza, porque al potenciar el cambio de actitudes es posible la subsecuente modificación de conductas y hábitos adquiridos que, al ponerse en contacto con otros miembros de la comunidad, propiciará patrones de enseñanza e imitación de actitudes pro-ambientales. Por último, se reconoce que la sobrepoblación es uno de los problemas ambientales álgidos en el mundo contemporáneo. Al respecto resulta sorprendente comparar la respuesta de los niños y niñas con el aporte del trabajo de Castanedo (1995) que 
encontró que el $51 \%$ de las personas están en desacuerdo o muy en desacuerdo frente a que "debería existir una ley para evitar el crecimiento desmesurado de la población".

En cuanto a las causas naturales asociadas a los problemas referidos al agua, se privilegian situaciones climáticas como el calentamiento global y el verano, entendido, según los participantes, como el exceso de calor por el sol. Es preciso notar que estas causas son producto de observaciones iniciales de fenómenos como las sequías o las inundaciones, y es en este punto donde se deben hacer distinciones entre lo que es una causa y lo que se define como consecuencia de un problema ambiental.

Resulta sumamente interesante el hecho de que la falta de gestión y responsabilidad comunitaria y gubernamental sea un factor que refleje preocupación entre los participantes, y resulta importante en la medida en que se reconoce que la responsabilidad de cuidado del agua radica no solo en las personas que consumen a diario el agua, sino también en los gobiernos encargados de su gestión. Estas respuestas coinciden con el trabajo de Castanedo (1995), en el cual frente a la responsabilidad gubernamental cerca del $95 \%$ de los participantes consideran que "el gobierno debería introducir medidas severas para frenar la contaminación ambiental".

En cuanto a la categoría Alternativas de conservación hacia el agua, se observó fuertes tendencias que instaban separadamente por los procesos educativos y por las soluciones tecnológicas como estrategias de conservación. Pensar que los saldos ambientales que deja el creciente modelo tecnológicodesarrollista se puedan solucionar con más tecnología, resulta algo paradójico. Según la Psicóloga ambiental Claude-Lévy Leboyer (citada por Zimmermann, 1995) los problemas ambientales son la resultante del avance técnico y las consecuencias que este trae: industrialización, explosión demográfica, consumismo, concentraciones humanas cada vez más alejadas de los valores culturales de sus pobladores ancestrales. Al respecto, Santamarina (citado por González y Figueroa, 2009), pone en entredicho los alcances de la Ciencia y la Tecnología como posibilidades de progreso. Castanedo (1995) tras la lectura del Informe Final de la Conferencia de Tbilisi, llega a la conclusión de que no es posible generar cambios sustanciales en los problemas ambientales haciendo uso únicamente de la tecnología. Es necesario incorporar valores y actitudes centrándose en la conducta que los individuos tienen con su entorno inmediato.

Ante el abismo separatista de las tendencias de Educación o Tecnología como alternativas de solución ante la crisis ambiental por el agua, Mayer (1998) propone que es necesario mediar en procura de buscar una homogeneidad entre escuela y tecnología, entre uso y consumo de los recursos. Ahora bien, de acuerdo con Lucie Sauvé (citada por Mayer, 1998) la pregunta es cómo aunar estas dos tendencias sin caer en lo que hoy por hoy está llamada a andar la educación; en un paradigma 
tecnológico donde se procura que la escuela esté al servicio de formar seres para la competitividad y la productividad.

En la categoría Toma de decisiones frente al aprovechamiento del agua, ciertamente los niños y niñas priorizan el suministro del agua en contraste con la construcción de la fábrica, que proveería de empleo a los habitantes de la ciudad. Los resultados coinciden con el Informe del MOPU (citado por Castanedo,1995), Recomendaciones para una Estrategia Nacional de Educación Ambiental en el Sistema Educativo, donde el $66,9 \%$ se declaraban en contra frente a la posibilidad de construir una fábrica altamente contaminante para los ríos y la atmósfera cerca a la casa, así proveyera de trabajo a un sinnúmero de personas. Incluso en los resultados de la investigación de Castanedo (1995) el 81,6\% se manifestaron en desacuerdo frente a la afirmación de que los beneficios que aportan los productos tecnológicos y de consumo son más importantes que la contaminación que resulta de la producción y uso de los mismos; y el 53,7\% se manifestaron de acuerdo en que debería evitarse la construcción de fábricas que contaminen los ríos y la atmósfera, aunque estas generen empleo.

Al cuestionar sobre los Actores responsables del cuidado del agua teniendo en cuenta que el sector agrícola gasta cerca del $70 \%$ del agua disponible para el consumo humano, las opiniones fueron divididas, sin embargo hubo una fuerte tendencia a atribuir dicha responsabilidad a los habitantes de la ciudad, en términos del mal uso del agua, y a justificar el gasto de agua en la zonas rurales debido al suministro alimentario que provee el campo a la ciudad.

Más allá de adelantar juicios sobre las responsabilidades de unos u otros, el análisis debe centrarse en una figura que parecía olvidada y es creer que el centro de la naturaleza es el ser humano $y$, por tanto, recae en él la responsabilidad del cuidado de los elementos que interactúan en la naturaleza. Esto no solo supone un antropocentrismo falsamente positivo, sino una condescendencia hacia el ambiente un tanto reprochable, en la medida en que de acuerdo con Mayer (1998) el ambiente natural no necesita de benefactores, al contrario, es la especie humana la que demanda urgentemente un entorno apto para vivir, garantizando la perdurabilidad de la misma.

En la categoría Agua como elemento esencial, al cuestionar a los participantes acerca de la idea de privilegiar la construcción de una carretera sobre un pozo de agua, facilitando el transporte de alimentos y con ello el aumento de los ingresos económicos, hubo una gran tendencia a darle prelación al agua sobre los ingresos económicos. Sin embargo, al leer el contexto, se observa que las razones para privilegiarla atienden a las incomodidades que sugiere tener que ir a otra fuente hídrica y con ello las implicaciones de costos de gasolina. Al priorizar el agua por el bienestar que genera, salta a la vista la incómoda verdad que se tiene de concebirla desde una visión recursista, al servicio de las comunidades y no como sistema dinámico en el que se desarrollan relaciones de co-dependencia. De igual 
manera, se tipifica que la comodidad se convierte en un fuerte estímulo para optar por la construcción de la carretera. Esto coincide con el trabajo de Berenguer y Corraliza (2000), donde el $51,5 \%$ de los encuestados revelan que emprenden acciones que saben que le perjudican al ambiente pero que les resultan cómodas, y el $88,3 \%$ opinan que para la gente es más importante su comodidad que el respeto hacia el ambiente.

En la categoría Agua como elemento de identidad, se obtienen, por una parte, respuestas favorables en torno a conservar el ecosistema de bosque y los sistemas de creencias alrededor de la laguna ancestral, y buscar soluciones prácticas para el transporte de agua a la comunidad aislada que no impliquen afectación. En la investigación de Castanedo (1995), se obtienen respuestas similares dado que en su escala, el $84,4 \%$ de los participantes aceptaban lo injusto que resulta deteriorar de la manera que actualmente se hace al considerar la naturaleza como un bien social. Sin embargo, por otro lado se obtienen respuestas que se engloban en una subcategoría denominada antropocentrismo del agua, esto es, privilegiar a toda costa el suministro de agua para el ser humano desconociendo, en palabras de González y Figueroa (2009), "los derechos intrínsecos" que tienen todas las formas de vida. No se trata entonces de imponer una visión romántica conservacionista sino, según los autores, de adquirir una ética ecocentrista donde se reconozca que todos los elementos que conforman el ambiente son sujetos de derecho, y se promueva el respeto de las relaciones de co-dependencia que entre ellos se establecen.

En las categorías Agua como elemento esencial y de identidad, se observa una situación particular, en la que si bien la mayoría de participantes reflejan actitudes pro-ambientales hacia el agua, no se es tan radical como en las otras respuestas del cuestionario y también se contempla la idea de privilegiar ingresos económicos, al construir la carretera, y de pasar el tubo por el bosque, para proveer de agua a la comunidad. Siguiendo a Castanedo (1995), esto radica en que cuando las medidas que se desean tomar afectan los estilos de vida, las respuestas que se consideran socialmente aceptadas, disminuyen drásticamente.

También es de notar que entre la subcategoría consecuencias de las actuaciones, los sentimientos de culpa y responsabilidad afloran. Al respecto Páramo y Gómez (1997) afirman que estos son muestras de una dimensión afectiva fuertemente establecida hacia el objeto de actitud, tipificándose dentro de lo que se conoce como preocupación ambiental que, en palabras de Berenguer y Corraliza (2000), es un parámetro para valorar el nivel de concienciación y una variable que predice futuros comportamientos ambientales.

Son varios los aportes que provee el análisis de la categoría Percepción afectiva del agua. Por un lado, para los participantes la importancia del agua radica en el uso que hacen de la misma en las actividades diarias. Lo que concuerda con las definiciones de servicio y recurso que se otorgan al agua. Lo anterior se 
corresponde claramente con las respuestas dadas en las otras categorías de análisis. Llama especialmente la atención, la connotación de reemplazable que se otorga al agua, más aún si se comprende que la vida tal como la conocemos no es posible sin el agua. En contraposición, se encuentran definiciones como tesoro, líquido preciado, que develan la valoración afectiva hacia el agua, y limitado donde, en palabras de González y Figueroa (2009), se observa que los participantes no caen en la falsa ilusión que fundamenta el modelo económico imperante, de creer que los recursos naturales son inagotables.

\section{AGRADECIMIENTOS}

Las autoras de la investigación expresan su agradecimiento a la Dirección de Investigaciones (DIN) de la Universidad Pedagógica y Tecnológica de Colombia y al Departamento Administrativo de Ciencia, Tecnología e Innovación, Colciencias, por la financiación del proyecto de investigación desarrollado durante la beca-pasantía otorgada en el marco de la Convocatoria No. 525: Programa Jóvenes Investigadores e Innovadores "Virginia Gutiérrez Pineda", Colciencias, 2012. De igual manera se agradece la iniciativa de todos los participantes que colaboraron en el proceso de investigación.

\section{REFERENCIAS}

- Aragonés, J. y Amérigo, M. (1991). Un estudio empírico sobre las actitudes ambientales. Revista de Psicología Social, 6(2), 223240.

- Berenguer, J. y Corraliza, J. (2000). Preocupación Ambiental y comportamientos ecológicos. Psicothema, 12, 325-329.

- Castanedo, S. (1995). Escala para la evaluación de las acittudes pro-ambientales (EAPA) de alumnos universitarios. Revista Complutense de Educación, 6, 253-278.

- Caurín, A. y Gil, Q. (2011). Las actitudes de los estudiantes universitarios ante la ciencia: métodos de análisis. Séptimo Congreso Internacional de Educación, Cultura y Desarrollo.

- Escudero, T. (1985). Las actitudes en la enseñanza de las ciencias: un panorama complejo. Revista de Educación, 278, 525.

- Gobernación de Boyacá. (15 de febrero de 2012). Acerca de: estado del recurso hídrico en Boyacá. Recuperado de http://www.gobernaciondeboyaca.gov.co

- González, E. y Figueroa, L. (2009). Los valores ambientales en los procesos educativos: realidades y desafíos. REICE, Revista Iberoamericana sobre Calidad, Eficacia y Cambio en Educación, 7(2), 95-115.

- Hernández, J. (2010). Cambio de actitudes y valores ante la energía tras el uso de un juego de rol. Teoría de la Educación. Educación y Cultura en la Sociedad de la Información, 11(3), 135148. 
- Iwatta, O. (1990). Relationship of Proenvironmental attitudes toward wildernism-urbanism and pro-preservation attitudes toward historical heritages. Psychologia, 33(4), 203-211.

- Mayer, M. (1998). Educación Ambiental: de la acción a la investigación. Enseñanza de las Ciencias, 2, 217-231.

- Pantoja, A. (2009). Manual básico para la realizacion de tesinas, tesis y trabajos de investigación. Madrid: Editorial EOS.

- Páramo, P. y Gómez, F. (1997). Actitudes hacia el medio ambiente: su medición a partir de la Teoría de Facetas. Revista Latinoamericana de Psicología, 29, 243-266.

- Secretaría de Educación de Boyacá. (2000). Competencias básicas en Ciencias Naturales y Pruebas de Estado. Boyacá.

- Sevillano, V. y Aragonés, J. (2009). Percepción social de la conducta de los españoles en materia medioambiental. Reis, Revista Española de Investigaciones Sociológicas, 126, 127-149.

- UNESCO. (1980). Educación Ambiental: las grandes orientaciones de la Conferencia Tbilisi. París: Centro de Publicaciones de la UNESCO.

- Van Liere, K. y Dunlap, R. (1981). Environmental Concern: Does it make a difference how it's measured? Environment and Behavior, 13(6), 651-676.

- Vargas, C. y Estupiñan, M. (2012). Estrategias para la Educación Ambiental con escolares pobladores del Páramo Rabanal (Boyacá). Luna Azul, 34, 10-25.

- Vargas, R. (2006). Cultura y democracia del agua. Polis, revista de la Universidad Bolivariana, 5(14).

- Weigel, R. y Weigel, J. (1978). Environmental Concern: The development of a measure. Environment and Behavior, 10(1), 315.

- Zimmermann, M. (1995). Psicología ambiental y calidad de vida. Colombia: ECOE Ediciones.

1. Artículo derivado de la investigación: "Agua Viva, un legado de nuestra cultura Muisca: estrategia didáctica para la consolidación de actitudes de compromiso, conocimiento y conservación hacia el agua", realizada durante la beca-pasantía otorgada por Colciencias en el marco del Programa Jóvenes Investigadores e Innovadores, y bajo la financiación de la Universidad Pedagógica y Tecnológica de Colombia, y el Departamento Administrativo de Ciencia, Tecnología e Innovación, Colciencias.

2. Licenciada en Ciencias Naturales y Educación Ambiental. Universidad Pedagógica y Tecnológica de Colombia. Grupo de Investigación GECOS. paolmend@hotmail.com

3. Magíster en Biología, Etología y Fisiología animal. Docente investigadora Licenciatura en Ciencias Naturales y Educación Ambiental. Universidad Pedagógica y Tecnológica de Colombia. Grupo de investigación GECOS. glolegu@yahoo.es 
Para citar este artículo: Mendieta-Hernández, M.P., GutiérrezGómez, G.L. (2014). Actitudes ambientales hacia el agua, una exploración en estudiantes del municipio de Ventaquemada (Boyacá). Revista Luna Azul, 39, 40-62. Recuperado de http://lunazul.ucaldas.edu.co/index.php?option=content\&tas k=view\&id=947 